\title{
Using Efficient RBF Network to Identify Interface Residues Based on PSSM Profiles and Biochemical Properties
}

\author{
Yu-Yen $\mathrm{Ou}^{1}$, Shu-An Chen ${ }^{1}$, Chung-Lu Shao ${ }^{2}$, and Hao-Geng Hung ${ }^{2}$ \\ ${ }^{1}$ Department of Computer Science and Engineering, \\ Graduate School of Biotechnology and Bioinformatics, \\ Yuan-Ze University, Chung-Li, Taiwan \\ 2 Department of Computer Science and Information Engineering, \\ National Taiwan University, Taipei, Taiwan
}

\begin{abstract}
Protein-protein interactions play a very important role in many biological processes, for example, information transfer along signaling pathways, and enzyme catalysis. Recently, scientists tried to predict the protein-protein interaction interface from sequences. Since the number of protein 3D structure still increase slowly comparing to the number of protein sequences, it may be a good idea to predict the protein-protein interface from sequences directly.

In this paper, the compositions and conserved functions of the amino acids in the protein interface are studied, and the information of secondary structures is added. In addition, we used radio basis function network to predict the protein interface with adding some useful biochemical features.
\end{abstract}

\section{Introduction}

Protein-protein interactions play a very important role in many biological processes, for example, information transfer along signaling pathways, and enzyme catalysis. Recently, scientists tried to predict the protein-protein interaction interface from sequences 12. Since the number of protein 3D structure still increase slowly comparing to the number of protein sequences, it may be a good idea to predict the protein-protein interface from sequences directly.

In [2], authors have developed a two-stage support vector machine (SVM) based method using amino acid sequence information to discriminate interface residues and non-interface residues from surface residues, and showed good results. In this paper, we try to use an efficient Radial Basis Function Network $(\mathrm{RBFN})$ classifier and PSSM profiles to enhance the prediction results. In addition, we adopt secondary structure information and some biochemical properties to improve the prediction accuracy. The experimental results showed that the additional information are useful for prediction.

The radial basis function network $(\mathrm{RBFN})$ is a special type of neural networks with several distinctive features 3456 . Since its first proposal, the RBFN has 
attracted a high degree of interest in research communities. An RBFN consists of three layers, namely the input layer, the hidden layer, and the output layer. The input layer broadcasts the coordinates of the input vector to each of the nodes in the hidden layer. Each node in the hidden layer then produces an activation based on the associated radial basis function. Finally, each node in the output layer computes a linear combination of the activations of the hidden nodes. How an RBFN reacts to a given input stimulus is completely determined by the activation functions associated with the hidden nodes and the weights associated with the links between the hidden layer and the output layer. The general mathematical form of the output nodes in an RBFN is as follows:

$$
c_{j}(x)=\sum_{i=1}^{k} w_{j i} \phi\left(\left\|x-\mu_{i}\right\| ; \sigma_{i}\right),
$$

where $c_{j}(x)$ is the function corresponding to the $j$-th output unit (class- $j$ ) and is a linear combination of $k$ radial basis functions $\phi()$ with center $\mu_{i}$ and bandwidth $\sigma_{i}$. Also, $w_{j}$ is the weight vector of class- $j$ and $w_{j i}$ is the weight corresponding to the $j$-th class and $i$-th center. The general architecture of RBFN is shown in Fig 1 .

In this paper, we select the spherical Gaussian function as our basis function of RBFN, so the Eq1 becomes:

$$
c_{j}(x)=\sum_{i=1}^{k} w_{j i} \exp \left(-\frac{\left\|x-\mu_{i}\right\|^{2}}{2 \sigma_{i}^{2}}\right) .
$$

From Eq2, we can see that constructing an RBFN involves determining the number of centers, $k$, the center locations, $\mu_{i}$, the bandwidth of each center, $\sigma_{i}$, and the weights, $w_{j i}$. That is, training an RBFN involves determining the values of three sets of parameters: the centers $\left(\mu_{i}\right)$, the bandwidths $\left(\sigma_{i}\right)$, and the weights $\left(w_{j i}\right)$, in order to minimize a suitable cost function.

Nevertheless, the essential task in constructing a RBFN classifier is to optimize the weights associated with the radial basis functions. In this paper, we proposes an efficient algorithm for determining the weights associated with the RBFN by exploiting the regularization theory [7] and the Cholesky decomposition [8]. The general observation is that the RBFN constructed is capable of delivering the same level of prediction accuracy as the SVM, while enjoying significant execution efficiency during the phase to construct the classifier.

\section{Constructing the Radial Basis Function Network}

In this paper, we focus on the calculation of the weights, so we conduct the simplest method to determine the centers and bandwidths. We have adopted all training instances as centers in the our experiments. Also, we employ the simplest method which is use the fixed bandwidth of each kernel function, and set the bandwidth as 5 for each kernel function. 


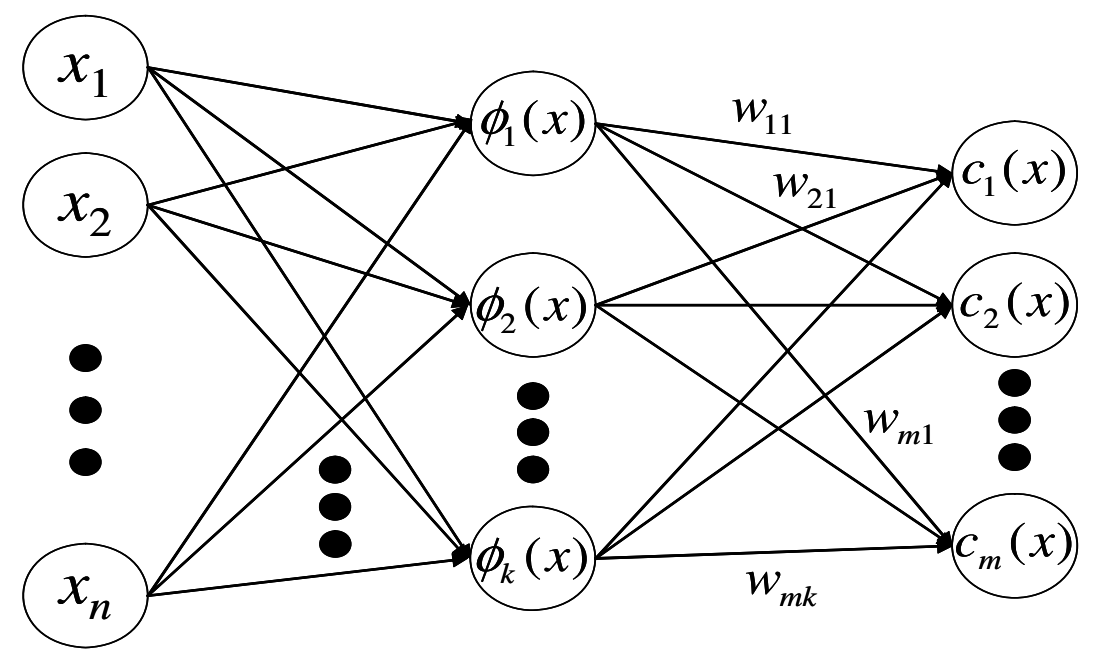

Input Layer

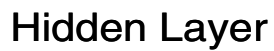

Output Layer

Fig. 1. General Architecture of Radial Basis Function Networks

After the centers and bandwidths of the kernel functions in hidden layer have been determined, the transformation between the inputs and the corresponding outputs of the hidden units is now fixed. The network can thus be viewed as an equivalent single-layer network with linear output units. Then, we use the lease mean square error (LMSE) method to determine the weights associated with the links between the hidden layer and the output layer.

In the following section, we will show how the LMSE method have been used in data classification field, and then propose a method which has a better theoretical foundation and practical use.

Assume $h$ is the output of the hidden layer.

$$
h=\left[\phi_{1}(x) \phi_{2}(x) \ldots \phi_{k}(x)\right]^{T},
$$

where $k$ is the number of centers, $\phi_{1}(x)$ is the output value of first kernel function with input $x$. Then, the discriminant function $c_{j}(x)$ of class- $j$ can be expressed by the following:

$$
c_{j}(x)=w_{j}^{T} h, j=1,2, \ldots, m
$$

where $m$ is the number of class, and $w_{j}$ is the weight vector of class- $j$. We can show $w_{j}$ as:

$$
w_{j}=\left[\begin{array}{llll}
w_{j 1} & w_{j 2} & \ldots & w_{j k}
\end{array}\right]^{T} .
$$

After calculating the discriminant function value of each class, we choose the class with the biggest discriminant function value as the classification result. We will discuss how to get the weight vectors by using least mean square error method in the following subsections. 


\subsection{Traditional Least Mean Square Error Method}

The traditional LMSE method was proposed by Broomhead and Lowe 9]. This method is originally proposed for function approximation, and is the most popular supervised learning method of constructing the weights of RBFN [4|5]10]11]. In this method, the objective function of class- $j$ can be shown as:

$$
\min \sum_{i=1}^{n}\left[c_{j}\left(x_{i}\right)-v_{j}\left(x_{i}\right)\right]^{2},
$$

where

$$
v_{j}\left(x_{i}\right)=\left\{\begin{array}{ll}
1 & \text { if } x \in \text { class }-j \\
0 & \text { otherwise }
\end{array} .\right.
$$

This system is overconstrained, being composed of $n$ equations with $k$ unknown weights, then the optimal solution of $w_{j}$ can be written as

$$
w_{j}^{*}=\Phi^{+} y_{j},
$$

where $y_{j}=\left[v_{j}\left(x_{1}\right) v_{j}\left(x_{2}\right) \ldots v_{j}\left(x_{n}\right)\right]^{T}, \Phi_{l i}=\phi_{i}\left(x_{l}\right)$ and $\Phi^{+}$is the pseudoinverse of $\Phi$. The matrix $\Phi$ is rectangular $(n \times k)$ and its pseudoinverse can be computed as

$$
\Phi^{+}=\left(\Phi^{T} \Phi\right)^{-1} \Phi^{T},
$$

provided that $\left(\Phi^{T} \Phi\right)^{-1}$ exists. The matrix $\left(\Phi^{T} \Phi\right)$ is square and its dimensionality is $k$, so that it can be inverted in time proportional to $k^{3}$.

The cost of computing $\Phi^{+}$is very high. Especially, we need to store $\Phi$ of size $(n \times k)$ in the memory. The value of $n$ in some classification problems is very large, such that it may be impractical to have such large amounts of memory space for storage. Therefore, this method may not be suitable for the use of classification problem.

\subsection{Least Mean Square Error Method with Statistics Techniques}

The improved LMSE method for data classification was proposed by Devijver et. al.[12. The idea of this method is basically the same with the traditional method, but 12 used the statistics techniques to analyze the whole problem. From this aspect, we can get the following results, and the major advantage from this approach is that we don't need to store $\Phi$ in the memory.

For a classification problem with $m$ classes, let $V_{i}$ designate the $i$-th column vector of an $m \times m$ identity matrix and $W$ be an $k \times m$ matrix of weights:

$$
W=\left[\begin{array}{llll}
w_{1} & w_{2} & \ldots & w_{m}
\end{array}\right] .
$$

Then the objective function to be minimized is

$$
J(W)=\sum_{j=1}^{m} P_{j} E_{j}\left\{\left\|W^{T} h-V_{j}\right\|^{2}\right\},
$$


where $P_{j}$ and $E_{j}\{\}$ are the a priori probability and the expected value of class- $j$, respectively.

To find the optimal $W$ that minimizes $J$, we set the gradient of $J(W)$ to be zero:

$$
\begin{aligned}
\nabla_{W} J(W) & =2 \sum_{j=1}^{m} P_{j} E_{j}\left\{h h^{T}\right\} W-2 \sum_{j=1}^{m} P_{j} E_{j}\{h\} V_{j}^{T} \\
& =[0],
\end{aligned}
$$

where [0] is a $k \times m$ null matrix.

Let $K_{i}$ denote the class-conditional matrix of the second-order moments of $h$, i.e.

$$
K_{i}=E_{i}\left\{h h^{T}\right\} .
$$

If $K$ denotes the matrix of the second-order moments under the mixture distribution, we have

$$
K=\sum_{j=1}^{m} P_{j} K_{j} .
$$

Then Eq. 11 becomes

$$
K W=M,
$$

where

$$
M=\sum_{j=1}^{m} P_{j} E_{j}\{h\} V_{j}^{T} .
$$

If $K$ is nonsingular, the optimal $W$ can be calculated by

$$
W^{*}=K^{-1} M .
$$

When compared to the traditional method, the size of $K, k \times k$, is much smaller than the $\Phi$ matrix of size $(n \times k)$ described in the previous subsection. Therefore, this method requires less memory space for storing the matrix.

However, there is a critical drawback of this method. That is, $K$ may be singular and this will crash the whole procedure. By observing the matrix $h h^{T}$, we are aware of that the matrix $h h^{T}$ is symmetric positive semi-definite (PSD) matrix with rank $=1$. Since $K$ is the summation of $h h^{T}$ for each training instance, $K$ is also a PSD matrix with rank $\leq n$. However, PSD matrix may be a singular matrix, so we should add the regularization term to make sure the matrix will be invertible.

In the regularization theory [7, it consists in replacing the objective function as follows:

$$
J(W)=\sum_{j=1}^{m} P_{j} E_{j}\left\{\left\|W^{T} h-V_{j}\right\|^{2}\right\}+\lambda \sum_{j=1}^{m} w_{j}^{T} w_{j},
$$

where $\lambda$ is the regularization parameter. Then the Eq. 14 becomes

$$
(K+\lambda I) W=M .
$$


If we set $\lambda>0,(K+\lambda I)$ will be a positive definite (PD) matrix and therefore is nonsingular. The optimal $W^{*}$ can be calculated by

$$
W^{*}=(K+\lambda I)^{-1} M \text {. }
$$

However, the PD matrix has many good properties, and one of them is a special and efficient triangular decomposition, Cholesky decomposition. By using Cholesky decomposition, we can decompose the $(K+\lambda I)$ matrix as follows,

$$
(K+\lambda I)=L L^{T},
$$

where $L$ is a lower triangular matrix. Then, the Eq. 18 becomes

$$
\left(L L^{T}\right) W=M \text {. }
$$

Actually, we can solve the linear system efficiently by using backsubstitution twice. In our experiments, Cholesky decomposition is about 10-20 times faster than alternative methods for matrix inversion. For example, in our experiments, we only used 25.36 seconds for inverting a $2558 \times 2558$ matrix in letter data set, while the traditional method used 754.93 seconds. It's about 30 times faster than the traditional method.

Finally, we can get the optimal $w_{j}^{*}$ for class- $j$ from $W^{*}$, and then the optimal discriminant function $c_{j}(x)$ for class- $j$ is derived. By using the regularization theory, the optimal weights can be obtained analytically and efficiently.

\section{Experimental Results of Interface Residues Prediction}

\subsection{Datasets}

We adapt the same dataset from Yan et al [2]. The dataset is originally from Chakrabarti et al. 13. Yan et al. selected 77 protein chains from 70 proteinprotein complexes.

\subsection{PSSM Profiles}

Recently, scientists try to use the Position Specific Scoring Matrix (PSSM) profiles as features in residues level function or structure prediction. [14 15] In this paper, we also adapt the PSSM profiles as our primal feature set instead of sequence residue type only. We obtain the PSSM profiles by using PSI-BLAST and non-redundant (NR) protein database. Also, every element has been scaled by $\frac{1}{1+e^{-x}}$.

\subsection{Biochemical Properties}

We tried 7 biochemical properties, which are hydrophobic, polar, small, aliphatic, aromatic, positive, and negative. In addition, we consider the properties toward interface-based or surface-based. That is, if the amino acid appears in interface more frequent than in surface, we think this amino acid is interface-based. Otherwise, we think the amino acid is surface-based. We list the properties with interface-based/surface-based in Table 1. 
Table 1. Amino Acid interface-based(I)/surface-based(S) Properties

\begin{tabular}{|c|c|c|c|c|c|c|c|c|c|c|c|c|c|c|c|c|c|c|c|c|}
\hline Prop & & L & V & C & A & $\mathrm{G}$ & M & $\mathrm{F}$ & Y & $\mathrm{W}$ & $\mathrm{H}$ & $\mathrm{K}$ & $\mathrm{R}$ & $\mathrm{E}$ & Q & $\mathrm{D}$ & $\mathrm{N}$ & S & & \\
\hline Iydrop & & & 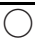 & $*$ & * & & & O & & O & O & * & O & O & O & & O & $\overline{0}$ & * & \\
\hline Hydro & & $*$ & * & & & & * & * & * & $*$ & $*$ & & O & $\overline{\mathrm{O}}$ & O & O & 0 & 0 & O & \\
\hline Pol & & & 0 & O & 0 & & 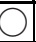 & 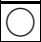 & O & 0 & 0 & $*$ & 0 & * & $*$ & * & * & * & $*$ & 0 \\
\hline$\overline{\mathrm{Pol}}$ & & & O & 0 & O & & & & & $*$ & * & & & & & O & 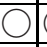 & 0 & O & \\
\hline Sma & & $\mathrm{O}$ & 0 & $*$ & O & $*$ & 0 & $\mathrm{O}$ & O & 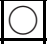 & O & O & O & O & O & $*$ & $*$ & $*$ & $*$ & * \\
\hline Sm & & $\mathrm{O}$ & & & $*$ & & & $\mathrm{O}$ & & $\mathrm{O}$ & O & O & O & O & O & $\mathcal{U}$ & & & & \\
\hline Aliph & & & & O & 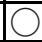 & & 0 & O & 0 & O & O & 0 & $O$ & 0 & & O & & 0 & & O \\
\hline Arom & & $\because$ & 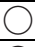 & O & 0 & $\triangle$ & O & $*$ & $*$ & $*$ & $*$ & O & O & O & O & O & O & O & O & \\
\hline Positi & & $\mathrm{O}$ & 0 & 0 & 0 & 0 & 0 & $\mathrm{O}$ & 0 & $\bigcirc$ & O & $*$ & O & O & O & O & O & 0 & O & $\mathrm{O}$ \\
\hline Posit & & 4 & 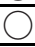 & $u$ & 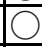 & 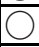 & 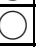 & $\mathrm{O}$ & ( & $\mathrm{O}$ & $*$ & 0 & $*$ & O & O & O & O) & 0 & O) & \\
\hline Negative_S & & & & & & & & & & & & & & $*$ & & $*$ & & & $\mathrm{OT}$ & \\
\hline
\end{tabular}

\subsection{Secondary Structure Information}

To further improve the prediction performance, we combined the PSSM profiles, biochemical properties and the predicted secondary structure from PSIPRED [14. Secondary structure play a very important role on protein folding and 3D structure, and generally believe that the function of protein is basically determined by its structure. We think the secondary structure information may useful on interface residues prediction. The experimental results show that the secondary structure information did improve the prediction performance.

\section{Results}

We used recall, precision, f-score, MCC (Matthew's correlation coefficient), ACC (accuracy) to measure the prediction performance. TP, FP, TN, FN are true positive number, false positive number, true negative number, and false negative number, respectively.

$$
\begin{gathered}
\text { Recall }=\frac{\mathrm{TP}}{\mathrm{TP}+\mathrm{FN}} \\
\text { Precision }=\frac{\mathrm{TP}}{\mathrm{TP}+\mathrm{FP}} \\
\mathrm{F} \text {-score }=\frac{2 \times \text { Recall } \times \text { Precision }}{\text { Recall }+ \text { Precision }} \\
\mathrm{ACC}=\frac{\mathrm{TP}+\mathrm{TN}}{\mathrm{TP}+\mathrm{FP}+\mathrm{TN}+\mathrm{FN}} \\
\mathrm{MCC}=\frac{\mathrm{TP} \times \mathrm{TN}-\mathrm{FP} \times \mathrm{FN}}{\sqrt{(\mathrm{TP}+\mathrm{FN})(\mathrm{TP}+\mathrm{FP})(\mathrm{TN}+\mathrm{FP})(\mathrm{TN}+\mathrm{FN})}}
\end{gathered}
$$

First of all, we compared our proposed RBF network classifier with Yan's results on 2. Mr. Yan provided us one of datasets of his experiment, and told us that the dataset may have some improper information within the training 
Table 2. Comparison results of Proposed Method and Yan Method

\begin{tabular}{lcccr}
\hline & $\begin{array}{c}\text { Proposed } \\
1^{\text {st }}\end{array}$ & \multicolumn{3}{c}{ Yan's Method 2 2] } \\
& stage $1^{\text {st }}$ & stage $2^{\text {nd }}$ & stage \\
\hline Precision & 0.42 & 0.77 & 0.44 & 0.58 \\
Sensitivity & 0.52 & 0.79 & 0.43 & 0.39 \\
Accuracy & 0.63 & 0.86 & 0.66 & 0.72 \\
MCC & 0.19 & 0.68 & 0.19 & 0.30 \\
\hline
\end{tabular}

Table 3. Comparison results of adding different biochemical properties

\begin{tabular}{lrrrrr}
\hline & Sensitivity & Precision Accuracy & \multicolumn{2}{c}{ MCC F-score } \\
\hline PSSM & 0.587 & 0.361 & 0.601 & 0.174 & 0.447 \\
\hline +Aliphatic_I & 0.594 & 0.374 & 0.616 & 0.197 & 0.459 \\
\hline +Aromatic_I & 0.597 & 0.379 & 0.621 & 0.204 & 0.463 \\
\hline +Positive_I & $\mathbf{0 . 6 0 8}$ & $\mathbf{0 . 3 7 5}$ & $\mathbf{0 . 6 1 4}$ & $\mathbf{0 . 2 0 1}$ & $\mathbf{0 . 4 6 4}$ \\
\hline +Small & 0.602 & 0.375 & 0.615 & 0.199 & 0.462 \\
\hline +hydrophobic & 0.599 & 0.378 & 0.619 & 0.203 & 0.463 \\
\hline +Negative_S & 0.591 & 0.378 & 0.620 & 0.201 & 0.461 \\
\hline +Polar_I & 0.599 & 0.377 & 0.618 & 0.202 & 0.462 \\
\hline
\end{tabular}

data. As Table 2 shows, our proposed classifier performs significantly better than results on 2, especially on second stage results. However, this dataset seems not fair on training and testing data, and than easily overfit with second stage process. We analyzed the problem, and concluded that the problem may be caused by residues based 5-fold cross validation, so we divided the 77 protein chains into 5 groups, and than used the new divided dataset as the comparison standard.

In Table 3, we listed the different results with the new divided dataset. The "PSSM" row listed the results by using PSSM profiles as features. Also, the following rows are the results by adding different biochemical properties as features. We can see the best results are from PSSM profile with 3 additional biochemical features, Aliphatic_I, Aromatic_I, and Positive_I.

In Table 4, we can see the secondary structure information can enhance the prediction accuracy. The final results show that the method proposed in this paper can achieve the 0.471 of F-score and 0.214 of MCC.

Table 4. Comparison results of different additional features

\begin{tabular}{lrrrrr}
\hline & Sensitivity & Precision & Accuracy & MCC F-score \\
\hline PSSM & 0.587 & 0.361 & 0.601 & 0.174 & 0.447 \\
PSSM+Biochemical & 0.608 & 0.375 & 0.614 & 0.201 & 0.464 \\
PSSM+Biochemical+SSE & 0.614 & 0.381 & 0.621 & 0.214 & 0.471 \\
\hline
\end{tabular}




\section{Conclusion}

In this paper, we proposed an efficient method to construct an RBFN classifier by using the improved LMSE method for constructing an RBFN optimized for data classification and bioinformatics applications. The method proposed by [12] is more efficient than the traditional one, but it may suffer the singular matrix problem and fails to build the classifier in such case. We solved the singular matrix problem by using the regularization theory, and used the Cholesky decomposition to speedup the matrix inversion process. This provides a good framework for constructing an RBFN in classification problems, and the proposed method can obtain the optimal weights analytically and efficiently.

We have applied our proposed approach to the prediction of interface residues. The interface residues prediction is one of the most important problems in computational biology and bioinformatics. Experimental results showed that combining proposed classifier and additional biochemical properties and secondary structure information can significant improve the prediction accuracy.

\section{References}

1. Zhou, H., Shan, Y.: Prediction of protein interaction sites from sequence profile and residue neighbor list. Proteins Structure Function and Genetics 44, 336-343 (2001)

2. Yan, C., Dobbs, D., Honavar, V.: A two-stage classifier for identification of proteinprotein interface residues. Bioinformatics 20, i371-i378 (2004)

3. Park, J., Sandberg, I.W.: Universal approximation using radial-basis-function networks. Neural Computation 3, 246-257 (1991)

4. Poggio, T., Girosi, F.: A theory of networks for approximation and learning. Technical Report A.I. Memo 1140, Massachusetts Institute of Technology, Artificial Intelligence Laboratory and Center for Biological Information Processing, Whitaker College (1989)

5. Ghosh, J., Nag, A.: An overview of radial basis function networks. In: Howlerr, R.J., Jain, L.C. (eds.) Radial Basis Function Neural Network Theory and Applications (2000)

6. Mitchell, T.M.: Machine Learning. McGraw-Hill, New York (1997)

7. Tikhonov, A.N., Arsenin, V.Y.: Solutions of Ill-Posed Problems. V.H. Winston \& Sons, John Wiley \& Sons, Washington D.C (1977)

8. Press, W.H.: Numerical Recipes in C, 2nd edn. Cambridge University Press, Cambridge (1992)

9. Broomhead, D.S., Lowe, D.: Multivariable functional interpolation and adaptive networks. Complex Systems 2, 321-355 (1988)

10. Orr, M.J.L.: Introduction to radial basis function networks. Technical report, Center for Cognitive Science, University of Edinburgh, UK (1996)

11. Tarassenko, I., Roberts, S.: Supervised and unsupervised learning in radial basis function classifiers. In: IEE Proceedings-Vision, Image and Signal Processing, vol. 141, pp. 210-216 (1994)

12. Devijver, P.A., Kittler, J.: Pattern recognition: a statistical approach. PrenticeHall, Englewood Cliffs (1982) 
13. Chakrabarti, P., Janin, J.: Dissecting protein-protein recognition sites. Proteins Structure Function and Genetics 47, 334-343 (2002)

14. Jones, D.T.: Protein secondary structure prediction based on position-specific scoring matrices. J. Mol. Biol. 292, 195-202 (1999)

15. Guo, J., Chen, H., Sun, Z., Lin, Y.: A novel method for protein secondary structure prediction using dual-layer svm and profiles. Proteins 54, 738-743 (2004) 